\title{
ZEROS AND PERIODICITY OF FUNCTIONS OF INFINITE MATRICES
}

\author{
by SHAFIK ASAAD IBRAHIM
}

(Received 27th May 1959)

\section{Introduction.}

Certain functions of infinite matrices are known to exist. $\dagger$ This gives rise to the following questions:

1. Whether the power series of matrices

$$
f(A)=\sum_{r=8}^{\infty} a_{r} A^{r}
$$

has a zero in the field $\ddagger$ of infinite matrices, and

2. If $f(A)$ exists for a certain infinite matrix $A$, is there an infinite matrix $B$ such that

$$
f(A+B)=f(A) ?
$$

In other words, is there a matrix period for $f(A)$ ?

In this paper theorems concerning zeros and periodicity of functions of semi block infinite matrices $§$ (defined below) are established.

\section{Definitions}

\section{Semi Block Infinite Matrices}

Let $S$ be the set of square matrices, including $1 \times 1$ matrices, and $\left\{S_{r}\right\}$ a sequence of matrices of $S$ of orders $w_{r}, r=0,1,2, \ldots$.

Let $A$ be an infinite matrix formed from the sequence $\left\{S_{r}\right\}$ arranged along its leading diagonal, and from arbitrary elements to the left, while all elements to the right of the $S_{r}$ are zero. We shall call such a matrix a lower semi block matrix.

The leading minors of $A$ of orders $w_{0}, w_{0}+w_{1}, w_{0}+w_{1}+w_{2}, \ldots$ form a sequence of square matrices $A_{r}$ each containing the previous members of the sequence, and having the property that all elements to the right of $A_{r}$ are zero. Given any element $a_{i j}$ of $A$, the first matrix in the sequence $A_{r}$ which contains $a_{i j}$ will be called the carrier of $a_{i j}$, and will be denoted by $A(i, j)$. The latent roots of $A_{r}$ are those of $S_{0}, S_{1}, \ldots, S_{r}$, since

$$
\operatorname{det}\left(A_{r}-\lambda I\right)=\prod_{i=0}^{r} \operatorname{det}\left(S_{i}-\lambda I\right) \text {. }
$$

We shall call every latent root of every matrix $S_{r}$ a scalar root of $A$; the set of all latent roots $\lambda_{i}$ of all the $S_{r}$ form the set of scalar roots of $A$.

$\dagger$ See, e.g., Cooke (1), 14; see also ibid., 38, Ex. 18, (ii), 270, Ex. 4, and Ibrahim (2).

† "Field" is not here used in the usual algebraic sense; see Cooke (1), p. 26, footnote.

$\S$ For further results about semi block infinite matrices, see Ibrahim (2) or (3).

II I am indebted to Dr P. Vermes for putting the definition in the present form, which is much shorter and clearer than mine. 
Upper semi block matrices are analogously defined. A lower semi block matrix $A$ will be of the form

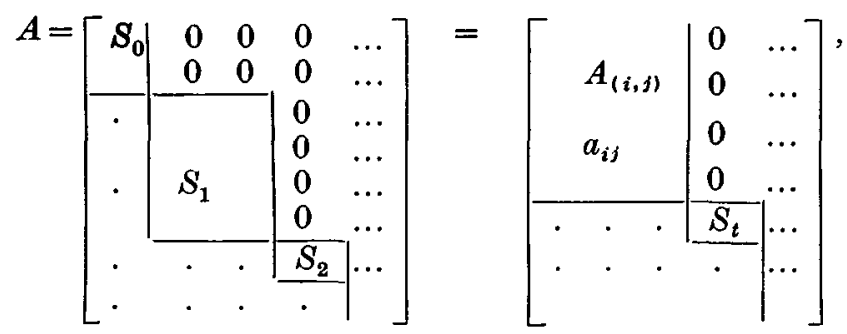

with arbitrary elements to the left of the $S_{r}$, giving

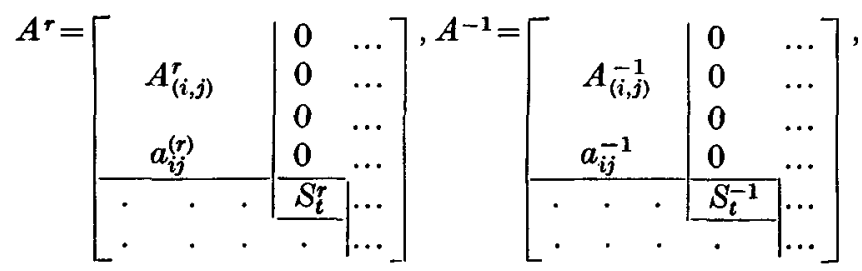

and generally for a function $f(A)$ of $A$,

$$
[f(A)]_{k, l}=[f\{A(k, l)\}]_{k, l} .
$$

Lemma 1. If $A$ is a lower (or upper) semi block matrix, then for every positive integer or zero (with $A^{\circ}=I$ ),

$$
\left(A^{r}\right)_{k, l}=\sum_{i=0}^{s}\left(A_{i, 0}+A_{i, 1} \frac{d}{d \lambda_{i}}+\ldots+A_{i, l_{i}-1} \frac{d l_{i}-1}{d \lambda_{i}^{l_{i}-1}}\right)_{k, l} \lambda_{i}^{r},
$$

where $\lambda_{i}(i=0,1,2, \ldots, s)$ are the latent roots of the matrix $A(k, l)$ the carrier of $a_{k, l}$, and $\lambda_{i}$ is repeated $l_{i}$ times, so that $\sum_{i=0}^{\varepsilon} l_{i}=n$, the order of the carrier $A(k, l)$. The

$$
A_{i, j}\left(i=0,1,2, \ldots, s ; j=0,1, \ldots, l_{i}-1\right)
$$

are polynomials in $A(k, l)$ of degrees $\leqslant n-1$.

Proof. As shown above, we have

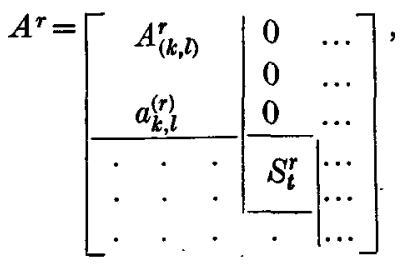

and since the carrier $A(k, l)$ satisfies the Hamilton-Cayley equation, the result follows.immediately by a simple transformation of Wedderburn's exposition. $\dagger$

$†$ See Wedderburn (4), 25-30; see also Cooke (I), 13. 
Remark. It is to be noted that the equation

$$
A^{r}=\sum_{i=0}^{8}\left(A_{i, 0}+A_{i, 1} \frac{d}{d \lambda_{i}}+\ldots+A_{i, l_{i}-1} \frac{d^{l_{i}-1}}{d \lambda_{i}^{l_{i}-1}}\right) \lambda_{i}^{r}
$$

without the suffixes $k, l$ on both sides has no meaning, since the left-hand side is an infinite matrix, whereas the right-hand side is a sum of finite square matrices.

Lemma 2. The exponential function exists for every lower (or upper) semi block matrix, and in particular for every lower semi-matrix. $\dagger$

Proof. By Lemma 1, and with its notation, we have

$$
\begin{aligned}
\left(e^{A}\right)_{k, l} & =\left[\sum_{r=0}^{\infty} \frac{A^{r}}{r !}\right]_{k, l}=\left[\sum_{r=0}^{\infty} \frac{A^{r}(k, l)}{r !}\right]_{k, l} \\
& =\sum_{i=0}^{g}\left(A_{i, 0}+A_{i, 1} \frac{d}{d \lambda_{i}}+\ldots+A_{i, l_{i}-1} \frac{d^{l_{i}-1}}{d \lambda_{i}^{l_{i}-1}}\right)_{k, l} \sum_{r=0}^{\infty} \frac{\lambda_{i}^{r}}{r !} \\
& =\sum_{i=0}^{8}\left(A_{i}\right)_{k, l} e^{\lambda i},
\end{aligned}
$$

where the $A_{i}$ are polynomials in $A(k, l)$ of degrees $\leqslant n-1$; i.e. $e^{A}$ exists for every semi block matrix $A$, and hence for the special case, namely, every lower semi-matrix.

(a) Zeros of functions of infinite matrices

Theorem 1. Let $A$ be a semi block infinite matrix with scalar roots and let $l_{i}$ be the multiplicity of $\lambda_{i}$.

$$
\lambda_{i}(i=0,1,2, \ldots) \text {, }
$$

Let $f(z)=\sum_{r=0}^{\infty} a_{r} z^{r}$ be convergent in a circle $D$; then $f(A)=0$ (the zero matrix) if the $\lambda_{i}$ are zeros of $f(z)$ with the same multiplicity $l_{i}$, and the $\lambda_{i}$ are all in $D$.

Proof. Since $\lambda_{i}$ is a zero of $f(z)$ of order $l_{i}$, we have

$$
f(z)=\left(z-\lambda_{i}\right)^{l} i \phi(z)
$$

where $\phi(z)$ is analytic in the neighbourhood of $z=\lambda_{i}$; also $\phi\left(\lambda_{i}\right) \neq 0$. This shows that

$$
f^{\prime}\left(\lambda_{i}\right)=f^{\prime \prime}\left(\lambda_{i}\right)=\ldots=f^{i_{i-1}}\left(\lambda_{i}\right)=0 .
$$

Now, by Lemma 1 , and with its notation, we have

$$
\begin{aligned}
{[f(A)]_{k, l} } & =\left[\sum_{r=0}^{\infty} a_{r} A^{r}\right]_{k, l} \\
& =\left[\sum_{r=0}^{\infty} a_{r} A^{r}(k, l)\right]_{k, l} \\
& =\sum_{i=0}^{8}\left[A_{i, 0}+A_{i, 1} \frac{d}{d \lambda_{i}}+\ldots+A_{i, l_{i}-1} \frac{d l_{i}^{l_{i}-1}}{d \lambda_{i}^{l_{i}-1}}\right]_{k, l} \sum_{r=0}^{\infty} a_{r} \lambda_{i}^{r} \\
& =\sum_{i=0}^{8}\left[A_{i, 0} f\left(\lambda_{i}\right)+A_{i, 1} f^{\prime}\left(\lambda_{i}\right)+\ldots+A_{i, l_{i}-1} f_{(\lambda i)}^{l_{i}-1}\right]_{k, l} \\
& =0 .
\end{aligned}
$$

This proves the theorem.

E.M.S.-P

† Compare Dienes' result, Cooke (1), 14, (1.7, I). 
Corollary. In particular, if the $\lambda_{i}$ are of multiplicity 1 , then $f(A)=0$ if the $\lambda_{i}$ are simple zeros of $f(z)$.

For example, the semi block infinite matrix $A$, whose scalar roots $\lambda_{1}$ are all distinct and are multiples of $2 \pi$ is such that $\sin A=0$ and $\cos A=I$.

It is clear that if $A$ is a semi block infinite matrix, then, by definition,

$$
\cos A=\sum_{r=0}^{\infty}(-1)^{r} \frac{A^{2 r}}{2 r !}, \sin A=\sum_{r=0}^{\infty}(-1)^{r} \frac{A^{2 r+1}}{(2 r+1) !}, \text { and } e^{A}=\sum_{r=0}^{\infty} \frac{A^{r}}{r !} ;
$$

and we easily see, as in Lemma 2 , that they all exist for every semi block infinite matrix. Also

$$
e^{i A}=\cos A+i \sin A \text { and } e^{-i A}=\cos A-i \sin A,
$$

i.e., $\quad \sin A=\frac{1}{2 i}\left(e^{i A}-e^{-i A}\right)$, and $\cos A=\frac{1}{2}\left(e^{i A}+e^{-i A}\right)$.

Again, $e^{A} \cdot e^{B}=e^{A+B}$, where $B$ is another semi block infinite matrix which commutes with $A . \dagger$

Hence

$$
\begin{aligned}
\sin ^{2} A+\cos ^{2} A & =-\frac{1}{4}\left(e^{2 i A}-2 I+e^{-2 i A}\right)+\frac{1}{4}\left(e^{2 i A}+2 I+e^{-2 i A}\right) \\
& =I .
\end{aligned}
$$

(b) Periodic Functions of Infinite Matrices

Definition. The function $f(A)$ of the infinite matrix $A$ is said to be periodic if there exists an infinite matrix $B$ such that

$$
f(A+B)=f(A)
$$

The infinite matrix $B$ is said to be the matrix period of $f(A)$.

Theorem 2. The matrix $i B$, where $B$ is a real semi block matrix whose scalar roots are all distinct and are multiples of $2 \pi$ is a period of the function $e^{A}$, where $A$ is another semi block matrix which commutes with $B$.

Proof. Since $A$ commutes with $B$, we have

$$
e^{A+i B}=e^{A} \cdot e^{i B}=e^{A}(\cos B+i \sin B) .
$$

But $\sin B=0$ and $\cos B=I$, as shown above. Therefore $e^{i B}=I$, and hence

$$
e^{A+i B}=e^{A} \cdot I=e^{A},
$$

which shows that $i B$ is a period of the function $e^{A}$.

Corollary. $B$, as defined in Theorem 2, is a matrix period of $\sin A$ and $\cos A$.

For, $e^{-i B}=\cos B-i \sin B=I$ and

$$
\begin{aligned}
\sin (A+B) & =\frac{1}{2 i}\left(e^{i A+i B}-e^{-i A-i B}\right) \\
& =\frac{1}{2 i}\left(e^{i A} \cdot e^{i B}-e^{-i A} \cdot e^{-i B}\right) \\
& =\frac{1}{2 i}\left(e^{i A}-e^{-i A}\right)=\sin A
\end{aligned}
$$

and similarly for $\cos A$. 
Finally, we remark that certain elliptic functions can be shown to exist for semi block infinite matrices.

\section{Acknowledgment}

I wish to acknowledge my sincere thanks to Dr R. G. Cooke for some remarks.

\section{REFERENCES}

(1) R. G. Cooke, Infinite Matrices and Sequence Spaces (Macmillan), 1950.

(2) S. A. Ibrahim (Ph.D. Thesis, University of London, 1955).

(3) S. A. Ibrahim, Structure of infinite matrices, J. London Math. Soc., 34 (1959), 281-288.

(4) J. H. M. Wedderburn, Lectures on Matrices, Amer. M.S. Colloquium Publications xvii (New York), 1934.

\section{Birkbeck College}

UNIVERSITY OF LONDON 